\title{
In situ X-ray diffraction studies on the piezoelectric response of PZT thin films
}

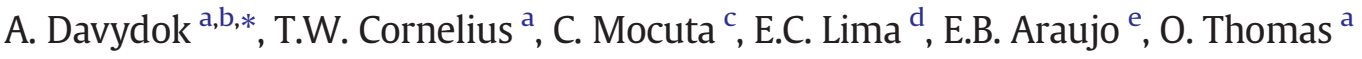 \\ a Aix Marseille Université, CNRS, Université de Toulon, IM2NP UMR 7334, 13397 Marseille, France

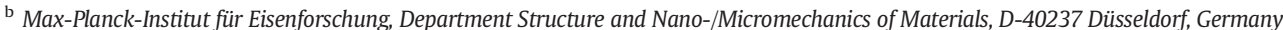 \\ c SOLEIL Synchrotron, DiffAbs beamline, L'Orme des Merisiers, Saint-Aubin - BP 48, 91192 Gif-sur-Yvette Cedex, France \\ d Universidade Federal do Tocantins, 77500-000 Porto Nacional, TO, Brazil \\ e Departamento de Fisica e Quimica, Universidade Estadual Paulista, Av. Brasil, 56 Centro, 15385-000 Ilha Solteira, SP, Brazil
}

\section{A R T I C L E I N F O}

\section{Article history:}

Received 5 October 2015

Received in revised form 21 January 2016

Accepted 22 January 2016

Available online 23 January 2016

\section{Keywords:}

Piezoeffect

X-ray diffraction

Thin film

Anisotropy

In-situ experiment

\begin{abstract}
A B S T R A C T
Piezoelectric properties of randomly oriented self-polarized $\mathrm{PbZr}_{0.50} \mathrm{Ti}_{0.50} \mathrm{O}_{3}$ (PZT) thin films were investigated using in situ synchrotron X-ray diffraction. Possibilities for investigating the piezoelectric effect using microsized hard X-ray beams are demonstrated and perspectives for future dynamical measurements on PZT samples with variety of compositions and thicknesses are given. Studies performed on the crystalline $[100,110]$ directions evidenced piezoelectric anisotropy. The piezoelectric coefficient $\mathrm{d}_{33}$ was calculated in terms of the lab reference frame ( $\left.d_{\text {perp }}\right)$ and found to be two times larger along the [100] direction than along the [110] direction. The absolute values for the $d_{\text {perp }}$ amount to 120 and $230 \mathrm{pm} / \mathrm{V}$ being in good agreement with experimental and theoretical values found in literature for bulk PZT ceramics.
\end{abstract}

(c) 2016 Elsevier B.V. All rights reserved.

\section{Introduction}

Lead zirconate titanate, $\mathrm{Pb}\left(\mathrm{Zr}_{1}-\mathrm{x}_{\mathrm{x}}\right) \mathrm{O}_{3}(\mathrm{PZT})$, is probably one of the most studied ferroelectric materials in the past years due to its excellent piezoelectric and dielectric properties [1]. The main studies on the PZT system were performed at compositions around the morphotropic phase boundary (MPB), at compositions of $x \approx 0.48$, where dielectric and piezoelectric properties show their maximum values. In thin film form, the potentials of PZT have been demonstrated for applications as capacitors [2], micromechanical devices [3], nonvolatile ferroelectric memories [4], and others.

The PZT system has been systematically investigated for several years to understand the high piezoelectric response at compositions around the MPB. The discovery of a stable ferroelectric monoclinic phase (space group $\mathrm{Cm}$ ) in the PZT system close to the MPB $[5,6]$ has provided a new perspective to explain some results attributed before to the coexistence of tetragonal (P4mm) and rhombohedral (R3m) phases $[7,8]$. Later, first-principles calculations and experimental findings were consistent with the monoclinic phase as an intermediate phase in a narrow region of composition between tetragonal and rhombohedral phases in the PZT system [8]. Although the high piezoelectric response could be explained in terms of a rotation of the polarization

\footnotetext{
* Corresponding author at: Max-Planck-Institut für Eisenforschung, Departmen Structure and Nano-/Micromechanics of Materials, D-40237 Düsseldorf, Germany.

E-mail address: davydok@mpie.de (A. Davydok).
}

from [001] to [111] directions in cubic indexes in the PZT [8], it is not yet clear if a monoclinic unit cell is a necessary condition for large piezoelectric responses or if the rotation of the polarization model really describes the origin of the higher piezoelectric responses in PZT and similar systems with MPB regions [9]. Despite the many uncertainties and controversies on this subject, it is unquestionable that our understanding of the piezoelectric response in perovskites has improved considerably in the last decade.

Although most of the studies in the past were on bulk ceramics, recent studies on PZT and other ferroelectric thin films have shown that the relaxation of a residual strain in films is accompanied by systematic changes in polarization properties [10]. Sometimes, these changes manifest as a self-polarization in thin films attributed to mechanical coupling between the ferroelectric film and a rigid substrate [11], to compressive/tensile stresses [12] or to Schottky barriers located at the bottom film-electrode interface $[13,14]$. These studies demonstrate an intrinsic dependence on sample size in ferroelectrics and have motivated studies on ferroelectric thin films.

Different techniques are widely used to measure the piezoelectric coefficients of bulk ferroelectrics. However, for thin films the absolute displacement of the surface is extremely small in comparison to bulk materials being often out of the resolution limit of displacement probes. Therefore, methods with high strain sensitivity are desirable to obtain precise values of the piezoelectric coefficients for thin films. In this context, the normal load method and interferometric measurements of electric field-induced displacements have been often used to measure 
the effective piezoelectric coefficient in bulk ferroelectric and thin films $[15,16]$. In addition, strain and piezoelectric properties of ferroelectric thin films can be measured quantitatively using time-resolved in situ synchrotron X-ray diffraction (XRD) with the appropriate spatial resolution and the sample under an applied electric field [17-20]. In situ high-resolution XRD has been performed to study the switching behavior of $90^{\circ}$ domains in epitaxial tetragonal PZT thin films under applied bias voltage [21-24], as well as to investigate the electromechanical properties and the origin of the polarization fatigue in highly (111) and (001) oriented tetragonal PZT thin films [25,26].

Recently, we observed a self-polarization effect $[27,28]$ on polycrystalline randomly oriented PZT thin films prepared by a modified Pechini polymeric method [29]. Although structural, electrical and local piezoelectric properties of these films were investigated to infer about the origin of this self-polarization effect [30], the piezoelectric anisotropy in this film was not yet investigated by in situ XRD. The present work reports on synchrotron microfocused X-ray diffraction experiments on such polycrystalline PZT thin films with a $\mathrm{Zr} / \mathrm{Ti}$ 50/50 concentration. The lattice expansion/contraction was monitored in-situ as a function of an applied electric field revealing "butterfly loops" [10] which are clear indications for the piezoelectric hysteresis. In addition, studies as a function of the crystalline orientation demonstrated piezoelectric anisotropy within the thin film showing piezoelectric coefficients which are two times larger along the [100] direction than along the [110] direction. The absolute values of the $d_{\text {perp }}$ amount to 120 and $230 \mathrm{pm} / \mathrm{V}$ for (100) and (110) oriented grains being in good agreement with experimental and theoretical values found in literature for bulk PZT.

\section{Experimental}

PZT thin films with a nominal composition $\mathrm{Pb}\left(\mathrm{Zr}_{0.50} \mathrm{Ti}_{0.50}\right) \mathrm{O}_{3}$ were deposited by spin coating on the $\mathrm{Pt} / \mathrm{TiO}_{2} / \mathrm{SiO}_{2} / \mathrm{Si}$ substrates using a polymeric precursor route based on the Pechini method [18]. As starting material, this method used PZT powder previously prepared by the conventional solid-state reaction as described in the literature [19]. For preparation of PZT powder, appropriate amounts of reagent-grade $\mathrm{PbO}$ (Aldrich, 99.9\%), $\mathrm{ZrO}_{2}$ (Aldrich, 99.9\%) and $\mathrm{TiO}_{2}$ (Aldrich, 99.99\%) were mixed at $\mathrm{Zr} / \mathrm{Ti}=50 / 50$ ratio by ball milling for $4 \mathrm{~h}$. The mixed powder was calcined at $850{ }^{\circ} \mathrm{C}$ for $3.5 \mathrm{~h}$ in an electric furnace to obtain the $\mathrm{PbZr}_{0.50} \mathrm{Ti}_{0.50} \mathrm{O}_{3}$ powder. Next, to prepare the polymeric resin PZT powder was dissolved in nitric acid solution $\left(30 \%\right.$ of $\mathrm{HNO}_{3}$ and $70 \%$ of distillate water) during $1 \mathrm{~h}$ at $80{ }^{\circ} \mathrm{C}$ to obtain the metallic $\mathrm{Pb}^{2+}, \mathrm{Zr}^{4+}$, and $\mathrm{Ti}^{4+}$ ions in solution. To compensate the lead loss during the film synthesis and to stabilize the growth of the perovskite phase, $10 \mathrm{~mol} \%$ of lead oxide was added in excess during the powder dissolution. After completing the dissolution, citric acid (CA) was added to the metal solution followed by stirring and heating at $90{ }^{\circ} \mathrm{C}$ for $1 \mathrm{~h}$ to form a citrate solution. Finally, ethylene glycol was added to the citrate solution and stirred while heating at around $110^{\circ} \mathrm{C}$ until a clear polymer was obtained. The obtained polymeric resin was deposited on the substrates by spin coating at $5000 \mathrm{rpm}$ for $30 \mathrm{~s}$. To remove organics, the polymer film was submitted to a pyrolysis at $300{ }^{\circ} \mathrm{C}$ for $30 \mathrm{~min}$. To increase the film thickness, another resin deposition and pyrolysis steps were performed. When the desired thickness was reached, the final film was obtained after a crystallization at $700^{\circ} \mathrm{C}$ for $1 \mathrm{~h}$. The obtained film was $710 \mathrm{~nm}$ in thickness and showed no preferential orientation of the grains. The film thickness was measured by scanning electron microscopy (SEM). For electrical measurements, several gold electrodes $(0.3 \mathrm{~mm}$ in diameter) were deposited by dc sputtering over an area of $1 \times 1 \mathrm{~cm}^{2}$ on the films through a mask to form capacitors. To apply an electric field, one of the gold electrodes was contacted using a thin wire with $150 \mu \mathrm{m}$ in diameter, as shown in the optical image presented as inset in Fig. 1(a). The DC voltage was applied using a Synchroneous Analog Output (SAO) card (model PXI 3 U from ADLINK), allowing to generate a bipolar tension in the $\pm 10 \mathrm{~V}$ range ( $5 \mathrm{~mA}$ maximum current).
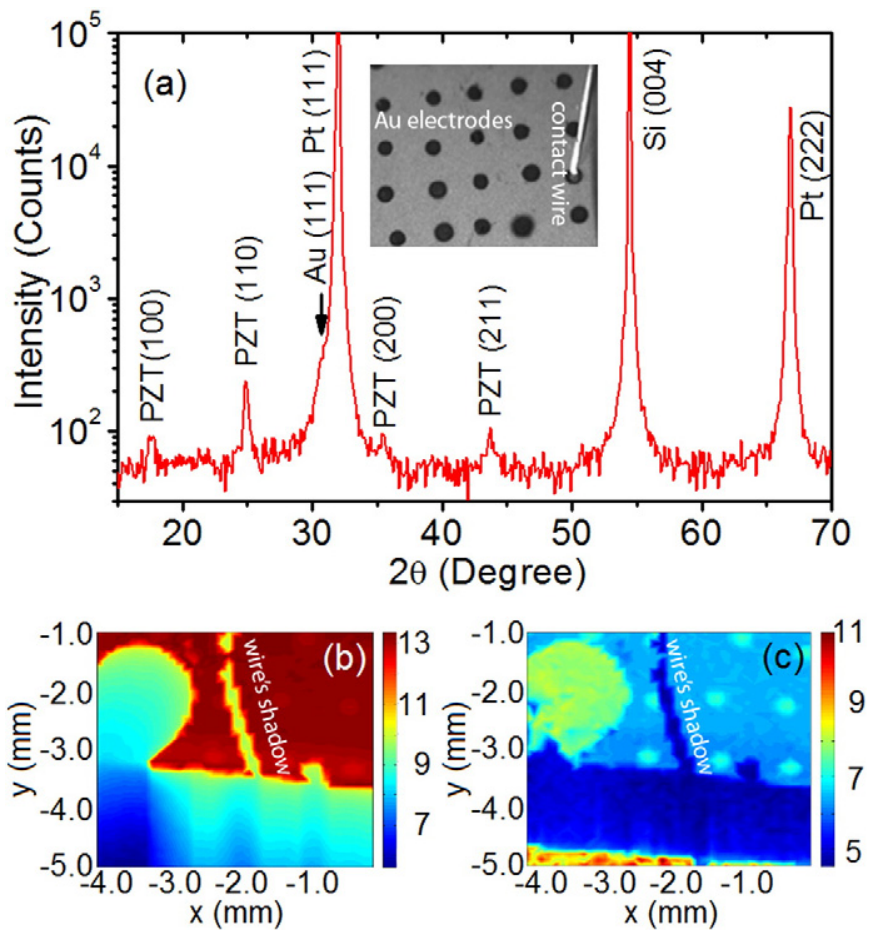

Fig. 1.a) $\theta / 2 \theta$ scan $(\lambda=1.2398 \AA$ ) in a large angular range covering Bragg reflections from all material components of the sample: PZT thin film deposited on the $\mathrm{Pt}(111) / \mathrm{TiO}_{2} / \mathrm{SiO}_{2} /$ Si substrate. The inset shows an optical microscopy image of the sample including the electrical connection via a thin wire. Scanning X-ray diffraction maps recorded $\mathrm{b}$ ) at the Pt 111 and c) at the Au 111 Bragg peaks.

In-situ X-ray diffraction (XRD) studies were performed at the DiffAbs beamline of SOLEIL Synchrotron (France) which is equipped with a 6 circle diffractometer (kappa geometry). The incident $10 \mathrm{keV}$ X-ray beam was focused down to a spot size of $8 \mu \mathrm{m} \times 5 \mu \mathrm{m}$ (full width half maximum, FWHM) in horizontal and vertical direction, respectively, using bent mirrors in Kirkpatrick-Baez (KB) geometry. This micrometric focal spot ensured that the footprint of the X-ray beam on the sample surface was always significantly smaller than the size of the top electrodes even at low incident angles of $\sim 8^{\circ}$ (footprint $\sim 60 \mu \mathrm{m} \times 5 \mu \mathrm{m}$ during the XRD measurements reported here). This configuration guarantees experiments in the central part of the electrodes where a homogeneous electric filed is expected and edge effects are avoided. The in situ diffraction measurements were performed in coplanar geometry with the sample mounted horizontally on a xyz translation-stage for precise lateral sample positioning. The diffracted intensity was monitored using a two-dimensional pixelated area detector (XPAD) with $560 \times 960$ pixels and a pixel size of $130 \mu \mathrm{m}$ [31]. It was installed at a distance of $650 \mathrm{~mm}$ from the sample covering a $6^{\circ}$ range in angular $2 \theta$ direction in a single image.

\section{Results and discussion}

A $\theta / 2 \theta$ diffractogram of the sample is presented in Fig. 1(a) revealing Bragg peaks of the PZT thin film, the Pt bottom electrode and the Si substrate. The Si 004 reflection served for alignment and as a reference for indexation purposes of the other Bragg peaks. The shoulder towards lower diffraction angles at the Pt 111 Bragg peak (highlighted by an arrow) originates from the gold electrodes deposited on top of the film.

In order to image the different sample regions and, in particular, to locate the contacted electrode, the sample was mapped over a range of $4 \times 4 \mathrm{~mm}^{2}$ in steps of $100 \mu \mathrm{m}$ using two different diffraction Bragg peaks as contrast signal for the maps. Fig. 1(b) and (c) displays scanning X-ray diffraction maps (SXDM) recorded using the Pt 111 and Au 111 Bragg peaks as contrast signal. While the position of the gold electrodes 
appear as less intense areas for the map at the Pt 111 Bragg reflection because of the absorption of the X-ray beam, they appear bright in the Au 111 SXDM. The extended shadow corresponds to the thin wire used for electrically contacting one of the Au electrodes which is located at the upper edge of the SXDMs. The extension of the shadow of the wire originates from the fact that the wire may block either the incident X-ray beam or the diffracted one during the raster mapping. After locating the electrically contacted Au electrode, the sample was laterally positioned in the X-ray beam in order to illuminate a region close to the contacting wire; then an electrical field was applied, and the diffraction intensity at the chosen PZT 110 and 100 Bragg peaks (cubic indexation) was recorded in-situ. Typical detector images after averaging 10 diffraction patterns which were recorded with an exposure time of $20 \mathrm{~s}$ each are shown in Fig. 2(a), for different applied potentials. All diffraction patterns show a segment of the Debye-Scherrer ring revealing a polycrystalline nature of the PZT thin film. With increasing electric field, the diffraction signal moves by few pixels on the detector, the maximum diffraction intensity decreases, and the width of the diffraction peak increases. For further data analysis, the XPAD images recorded for one applied electric field were averaged. Before image analysis geometrical corrections were applied on the curved signal stemming from the powder ring transforming the pixel coordinates of the detector into angular one (two theta and azimuth). The $2 \theta$ Bragg peak profile is extracted following an azimuthal regrouping of the data (pixels corresponding to the same $2 \theta$ value). Two such line profiles for an applied potential of $0 \pm 0.1 \mathrm{~V}$ and $9 \pm 0.1 \mathrm{~V}$ are shown in Fig. 2(b) respectively. On the one hand, the width of these line profiles allows for determining the average size of the grains constituting the film. The mean grain size amounts is about $20 \mathrm{~nm}$ which is in good agreement with TEM studies (not shown here). On the other hand, these line profiles verify the shift of the Bragg peak as a function of the applied voltage as well as the slight decrease at maximum intensity. In order to extract the position and the full width at half maximum of the Bragg peak, the line profiles were fitted by a Gaussian. While 1 pixel on the detector corresponds to an angular opening of $0.011^{\circ}$, the aforementioned fitting process allows for measuring sub-pixel shifts rendering an estimated strain resolution of the order of $10^{-4}$.

Fig. 2(c) presents the $2 \theta$ value of the fitted center position of the PZT 110 Bragg peak for $U=0 \mathrm{~V}$ and $U=9 \mathrm{~V}$ as a function of time. The exposure time for each diffraction pattern (i.e. detector image) was $20 \mathrm{~s}$. As soon as a voltage of $9 \mathrm{~V}$ is applied on the film $(t=0 \mathrm{~s})$, the $2 \theta$-position of the Bragg peak shifts towards lower values by $0.02^{\circ} \mathrm{im}$ plying that strain is induced by the applied electric field. After $600 \mathrm{~s}$, the voltage was switched back to the initial value of $U=0 \mathrm{~V}$. While maintaining the electric field, the Bragg peak continuously moves to lower angles with time, indicating a lattice expansion. This lattice expansion may originate from the Joule effect heating up the sample. Compared to the piezoelectric strain induced by the electric field, the thermal lattice expansion is a factor of 5 less over a time period of $10 \mathrm{~min}$. The studied sample contains Au and Pt electrodes whose thermal expansion coefficients are much higher than those for piezo ceramics [36] making them ideal as a reference for detecting heating effects. Hence, the evolution of the Pt 111 Bragg peak was studied as a function of the applied potential (not shown here) revealing a variation of $\sim 10^{-3}$ degrees in its $2 \theta$-position, which is close to the resolution limit of this experimental approach. This change in diffraction angle corresponds to a thermal strain of $<10^{-4}$ and, thus to a temperature increase of $<10^{\circ} \mathrm{C}$ at $\mathrm{U}=9 \mathrm{~V}$. Thus, the thermal expansion by Joule heating was neglected for the further studies.

The piezoelectric response of the PZT thin film was investigated for two different crystalline orientations (PZT(110) and PZT(100)) by increasing the applied voltage from $0 \mathrm{~V}$ to $+9 \mathrm{~V}$, then decreasing it to $-9 \mathrm{~V}$ and raising it back to $0 \mathrm{~V}$ (i.e. $E$ ranging from -140 to $+140 \mathrm{kV} / \mathrm{cm}$ ) in steps of $0.5 \mathrm{~V}$. At each step, 30 diffraction patterns with an exposure time of $20 \mathrm{~s}$ each were recorded. Before increasing the voltage to the following step, the voltage was reduced to $0 \mathrm{~V}$ for $200 \mathrm{~s}$ in order to minimize a possible heating by the Joule effect as described before. From the change of the $2 \theta$ position of the center of the respective Bragg peak at $0 \mathrm{~V}$ and at the applied potential, the strain induced by the piezoelectric effects was calculated. Fig. 3(a) and (b) (a)

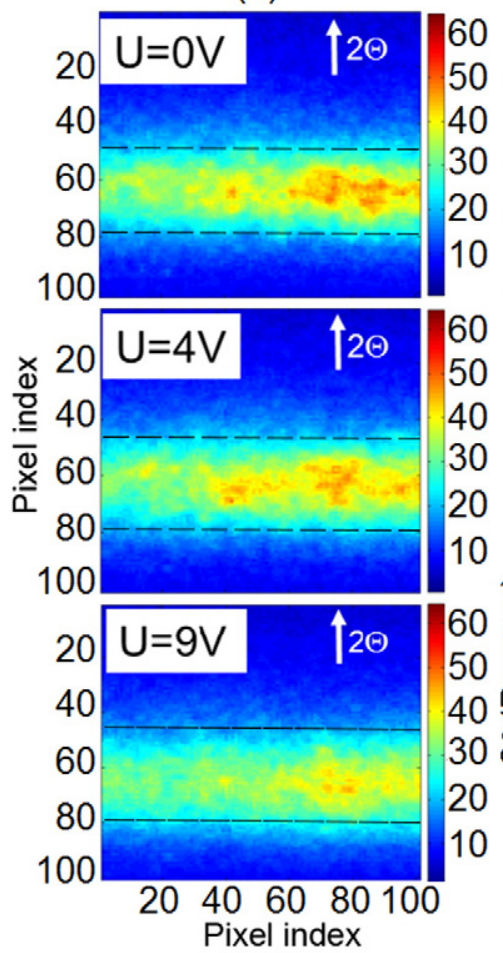

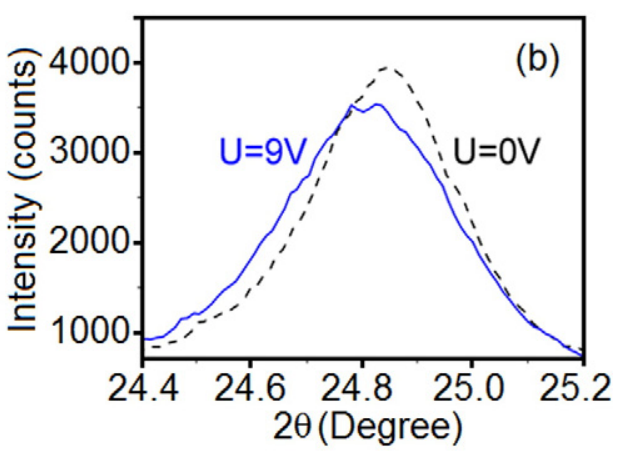

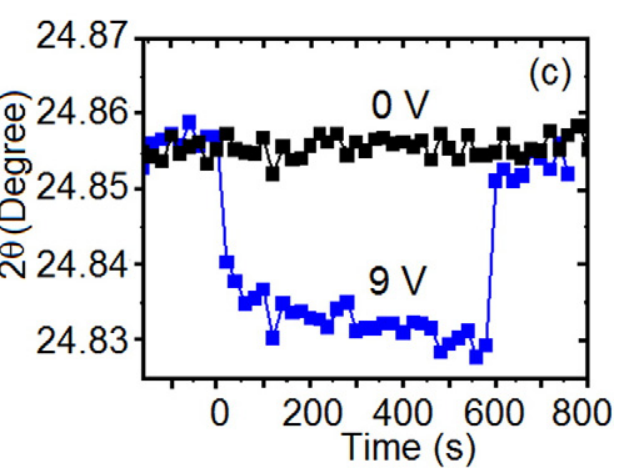

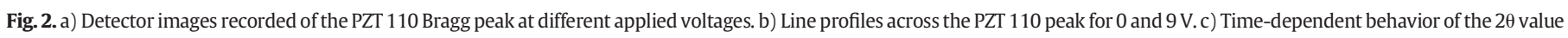
of the PZT 110 Bragg peak at $U=0 \mathrm{~V}$ and $\mathrm{U}=9 \mathrm{~V}$. 


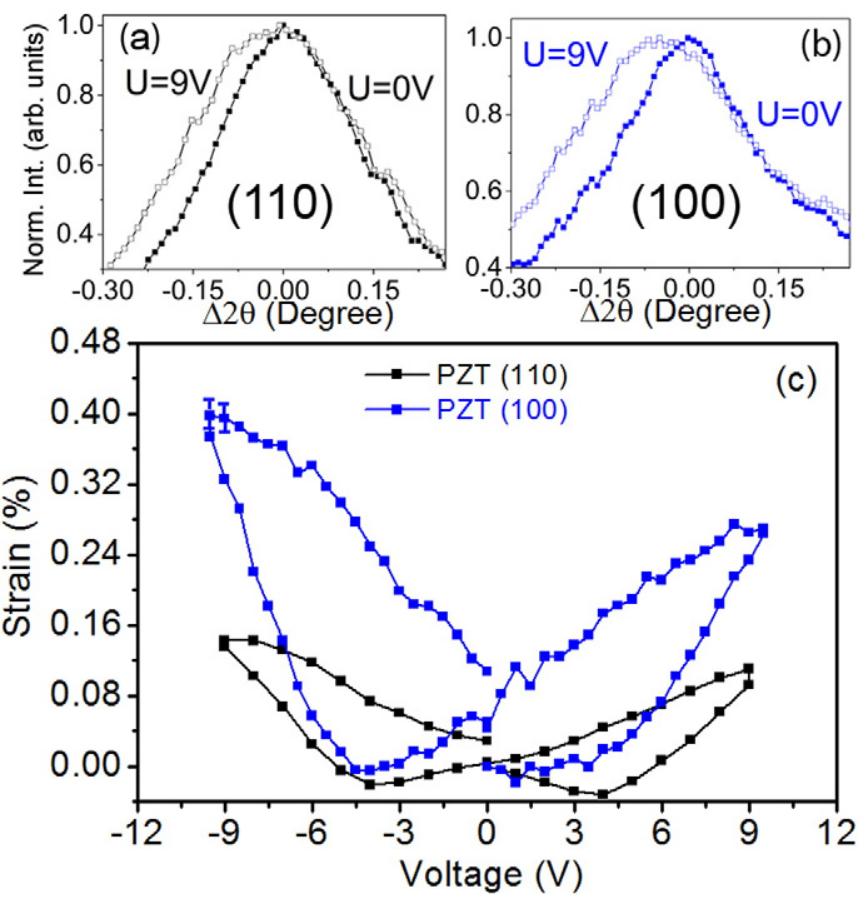

Fig. 3. Two-theta line profiles of a) the PZT 100 and b) the PZT 110 diffraction peak for applied voltages of $0 \mathrm{~V}(\boldsymbol{\square})$ and at $9 \mathrm{~V}(\square)$. c) Piezoelectric strain as a function of the applied electric potential for the PZT 110 and the PZT 100 Bragg reflections. The error bars originate from the uncertainty of the fitting of the profile of the Bragg peak using a Gaussian.

displays the $2 \theta$ profiles for the PZT 110 and 100 reflections, respectively, recorded at $U=0 \mathrm{~V}$ and $\mathrm{U}=9 \mathrm{~V}$. Based on those findings, strain profiles for the PZT (110) and the PZT (100) were calculated. Fig. 3(c) displays the piezoelectric strain for the two PZT reflections as a function of the applied voltage, revealing "butterfly loops" [32]. These loops are a clear signature for a piezoelectric hysteresis in the thin film. The piezoelectric effect is a factor of $\sim 2$ stronger for grains oriented with [100] out-of-plane direction than for grains oriented with [110] out-of-plane direction, evidencing piezoelectric anisotropy. Considering a linear voltage drop over the entire film thickness of $710 \mathrm{~nm}$, the piezoelectric coefficient in the lab reference frame $\mathrm{d}_{\text {perp }}$ at $U=9 \mathrm{~V}$ for the PZT (100) and PZT (110) yields $\sim 230$ and $\sim 110 \mathrm{pm} / \mathrm{V}$, respectively. These values are in good agreement with theoretical piezoelectric coefficients found in literature for these two crystalline orientations [33,34].

Both "butterfly loops" are characterized by an asymmetric shape and openings at $U=0 \mathrm{~V}$ (Fig. 3(c)). Such behavior was observed and explained for different materials including different compositions of PZT $[21,26,35,36]$. The asymmetry of the loops may originate from an uncomplete switching of the polarization domains [26] or from a selfpolarization of the film [13]. The latter may be caused by space charges associated to Schottky barriers located at the bottom film-electrode interface [37], complex defects such as lead and oxygen vacancies [37], or by the mechanical coupling of the film and the substrate resulting in compressive/tensile stress [11]. In the case of PZT films previous studies demonstrated that Schottky barriers can be ruled out as the main mechanism for the self-polarization of PZT thin films.

For the PZT film under study in the present work, asymmetries of the macroscopic P-E hysteresis loops and the analysis of the local piezoresponse by piezoresponse force microscopy indicated an internal bias electric field which is oriented towards the bottom electrode [29, 30]. This internal electric bias field (not estimated) which is a signature of the self-polarization of the film interferes with the applied extrinsic electric field ( $\max \pm 127 \mathrm{kV} / \mathrm{cm}$ ) resulting in an asymmetric effective electric field. Due to this superposition of the two electric fields the effective electric field is higher in the direction towards the bottom interface than towards the top surface. As a consequence, higher strains are observed for negative voltages compared to positive voltages leading to asymmetries in the measured butterfly curves (see Fig. 3(c)). While the effective field for negative applied voltages is sufficient to polarize the film in one direction, it is not clear if the magnitude of the effective electric field for positive applied voltages is large enough to overcome the positive coercive field $\mathrm{Ec}(+40 \mathrm{kV} / \mathrm{cm}$ and $-14 \mathrm{kV} / \mathrm{cm})$ and thus to switch the polarization of the studied film [29]. Further experiments are needed to uncover the origin of this behavior.

\section{Conclusions}

In conclusion, in-situ microfocused X-ray diffraction experiments on PZT thin films were conducted as a function of the applied electric field rendering possible both the direct measurement of the piezoelectric strain induced by the electric field and the determination of the piezoelectric coefficient for different crystalline orientations. Butterfly loops demonstrate the piezoelectric hysteresis in the material while the fact that the loops are not closed indicate the existence of a selfpolarization. The experiment further addresses the piezoelectric anisotropy: the piezoelectric coefficient is about a factor two larger in the [100] than in the [110] direction. These studies pave the way to novel research on the origin of the self-polarization effect in thin films as a function of their thickness and their composition. Moreover, future synchronous experiments in combination with AC electric fields may allow for dynamic measurements giving access to the reorientation of the piezoelectric domains.

\section{Acknowledgments}

The authors gratefully acknowledge the SOLEIL Synchrotron for allocating beam time. Detector and Electronics Support Groups, as well as P. Joly, are acknowledged for excellent technical support during the experimental campaign at SOLEIL Synchrotron on DiffAbs beamline. We would like to express our gratitude to the CAPES-COFECUB (Project $\mathrm{N}^{\circ}$ 801-14), CNPq (Research grant 305973/2012-6 and Project $\mathrm{N}^{\circ} 400677 / 2014-8$ ) and FAPESP (Project $\mathrm{N}^{\circ}$ 2010/16504-0) for their financial support.

\section{References}

[1] B. Jaffe, W. Cook, H. Jaffe, Piezoelectric Ceramics, Academic Press, New York, 1971

[2] M. Miyake, J.F. Scott, X.J. Lou, F.D. Morrison, T. Nonaka, S. Motoyama, T. Tatsuta, O. Tsuji, "Submicron three-dimensional trenched electrodes and capacitors for DRAMs and FRAMs: fabrication and electrical testing", J. Appl. Phys. 104 (2008) 064112 .

[3] C. Ahn, K. Rabe, J.,. Triscone, Ferroelectricity at the nanoscale: local polarization in oxide thin films and heterostructures“, Science 303 (2004) 488

[4] J. Scott, C. de Araujo, Ferroelectric memories, Science 246 (1989) 1400.

[5] B. Noheda, D.E. Cox, G. Shirane, J. Gonzalo, L. Cross, S.-E. Park, "A monoclinic ferroelectric phase in the $\mathrm{Pb}(\mathrm{Zr} 1$ - xTix)O3 solid solution" appl, Phys. Lett. 74 (1999) 2059.

[6] B. Noheda, J. Gonzalo, L. Cross, R. Guo, S.-E. Park, D.E. Cox, G. Shirane, Tetragonal-tomonoclinic phase transition in a ferroelectric perovskite: the structure of PbZr0.52Ti0.4803, Phys. Rev. B 61 (2000) 8687.

[7] R. Guo, L.E. Cross, S.-E. Park, B. Noheda, D.E. Cox, G. Shirane, "Origin of the high piezoelectric response in PbZr1 - xTixO3, Phys. Rev. Lett. 84 (2000) 5423.

[8] L. Bellaiche, A. Garcia, D. Vanderbilt, Finite-temperature properties of $\mathrm{Pb}(\mathrm{Zr} 1$ - xTix)O3 alloys from first principles, Phys. Rev. Lett. 84 (2000) 5427.

[9] B. Noheda, D.E. Cox, Bridging phases at the morphotropic boundaries of lead oxide solid solutions, Phase Transit. 79 (2006) 5.

[10] J. Cillessen, M. Prins, R. Wolf, "Thickness dependence of the switching voltage in alloxide ferroelectric thin-film capacitors prepared by pulsed laser deposition”, J. Appl. Phys. 81 (1997) 2777.

[11] A. Gruverman, B. Rodriguez, A. Kingon, R. Nemanich, A. Tagantsev, J. Cross, M. Tsukada, "Mechanical stress effect on imprint behavior of integrated ferroelectric capacitors", Appl. Phys. Lett. 83 (2003) 728.

[12] V. Afanasjev, I. Pronin, A. Kholkin, "Formation and relaxation mechanisms of the self-polarization in thin ferroelectric films", Phys. Solid State 48 (2006) 1214.

[13] A. Kholkin, K. Brooks, D. Taylor, S. Hiboux, N. Setter, "Self-polarization effect in $\mathrm{Pb}(\mathrm{Zr}, \mathrm{Ti}) \mathrm{O} 3$ thin films", Integr. Ferroelectr. 22 (1998) 525.

[14] V.Afanasjev, A. Petrov, I. Pronin, E. Tarakanov, E. Kaptelov, J. Graul, "Polarization and self-polarization in thin PbZr1-xTixO3 (PZT) films", J. Phys. Condens. Matter 13 (2001) 8755. 
[15] K. Lefki, G. Dormans, "Measurement of piezoelectric coefficients of ferroelectric thin films", J. Appl. Phys. 76 (1994) 1764.

[16] A. Kholkin, Ch. Wütchrich, D. Taylor, N. Setter, "Interferometric measurements of electric field induced displacement in piezoelectric thin films", Rev. Sci. Instrum. 67 (1996) 1935

[17] M. Ehmke, J. Glaum, M. Hoffman, J. Blendell, K. Bowman, In Situ X-ray Diffraction of Biased Ferroelastic Switching in Tetragonal Lead-free $(1-\mathrm{x}) \mathrm{Ba}(\mathrm{Zr0} 0.2 \mathrm{Ti0}$.8)O3x(Ba0.7Ca0.3)TiO3 Piezoelectrics“, J. Am. Ceram. Soc. 96 (2013) 2913.

[18] D. Carbone, A. Pateras, G. Bussone, P. Evans, T. Cornelius, M. Bousquet, A. Boulle, B. Gautier, J.R. Duclère, Structural observation of piezoelectric inhomogeneity in a mixed-orientation Na0.5Bi0.5TiO3 perovskite thin film, Appl. Phys. Lett. 105 (2014) 242901.

[19] H. Graafsma, G. Heunen, S. Dahaoui, A. El Haouzi, N. Hansen, G. Marnier, “The piezoelectric tensor element d33 of KTiOPO4 determined by single crystal X-ray diffraction", Acta Crystallogr. B 53 (1997) 565.

[20] O. Schmidt, S. Gorfman, L. Bohatý, E. Neumann, B. Engelen, U. Pietsch, Investigations of the bond-selective response in a piezoelectric Li2SO4. $\mathrm{H} 2 \mathrm{O}$ crystal to an applied external electric field, Acta Crystallogr. A 65 (2009) 267.

[21] K.S. Lee, Y. Kim, S. Baik, In situ observation of ferroelectric 90 degrees-domain switching in epitaxial $\mathrm{Pb}(\mathrm{Zr}, \mathrm{Ti}) \mathrm{O} 3$ thin films by synchrotron X-ray diffraction “, Appl. Phys. Lett. 79 (2001) 2444.

[22] S. Seshadri, A. Prewitt, A. Studer, D. Damjanovic, J. Jones, An in situ diffraction study of domain wall motion contributions to the frequency dispersion of the piezoelectric coefficient in lead zirconate titanate“, Appl. Phys. Lett. 102 (2013) 042911.

[23] D.-H. Do, A. Grigoriev, D. Kim, C.-B. Eom, P. Evans, E. Dufresne, In situ X-ray probes for piezoelectricity in epitaxial ferroelectric capacitors, Integr. Ferroelectr. 101 (2008) 174.

[24] V. Thery, A. Bayart, J.-F. Blach, P. Roussel, S. Saitzek, "Effective piezoelectric coefficient measurement of BaTiO3 thin films using the X-ray diffraction technique under electric field available in a standard laboratory", Appl. Surf. Sci. 351 (2015) 480 .

[25] N. Menou, C. Muller, I. Baturin, D. Kuznetsov, V. Shur, J. Hodeau, T. Schneller, In situ synchrotron X-ray diffraction study of electrical field induced fatigue in $\mathrm{Pt} /$ PbZr0.45Ti0.5503/Pt ferroelectric capacitors, J. Phys. Condens. Matter 17 (2005) 7681.
[26] D. Do, A. Grigoriev, D. Kim, C. Eom, P. Evans, E. Dufresne, In-situ X-ray probe of piezoelectricity in epitaxial ferroelectric capacitors, Integr. Ferroelectr. 101 (2009) 174.

[27] E.C. Lima, E.B. Araújo, I. Bidkin, A. Kholkin, The self-polarization effect in $\mathrm{Pb}(\mathrm{Zr0}$.50Ti0.50)O3 thin films with no preferential orientation, Mater. Res. Bull. 47 (2012) 3548.

[28] E.C. Lima, E.B. Araújo, A. Souza Filho, A. Paschoal, I. Bdikin, A. Kholkin, “Structural depth profile and nanoscale piezoelectric properties of randomly oriented Pb(Zr0.50Ti0.50)03 thin films", J. Phys. D. Appl. Phys. 45 (2012) 215304

[29] E.B. Araújo, J.A. Eiras, Structural, electric and ferroelectric properties of PZT films obtained using oxide precursors, J. Mater. Sci. Lett. 17 (1998) 833.

[30] E.B. Araújo, E.C. Lima, I.K. Bdikin, A.L. Kholkin, "Thickness dependence of structure and piezoelectric properties at nanoscale of polycrystalline lead zirconate titanate thin films", J. Appl. Phys. 113 (2013) 187206.

[31] C. Mocuta, M.-I. Richard, J. Fouet, S. Stanescu, A. Barbier, C. Guichet, O. Thomas, S. Hustache, A. Zozulya, D. Thiaudie're, "Fast pole figure acquisition using area detectors at the DiffAbs beamline - Synchrotron SOLEIL", J. Appl. Crystallogr. 46 (2013) 1842.

[32] J. Danielsa, T. Finlayson, A. Studer, M. Hoffman, J. Jones, "Time-resolved diffraction measurements of electric-field-induced strain in tetragonal lead zirconate titanate", J. Appl. Phys. 101 (2007) 094104.

[33] D. Taylor, D. Damjanovic, Piezoelectric properties of rhombohedral $\mathrm{Pb}(\mathrm{Zr}, \mathrm{Ti}) \mathrm{O} 3 \mathrm{~Pb}(\mathrm{Zr}, \mathrm{Ti}) 03$ thin films with (100), (111), and "random" crystallographic orientation, Appl. Phys. Lett. 76 (2000) 1615

[34] X. Du, U. Belegundu, K. Uchino, “Crystal orientation dependence of piezoelectric properties in lead zirconate titanate: theoretical expectation for thin films", Jpn. J. Appl. Phys. 36 (1997) 5580.

[35] J. Daniels, A. Pramanick, J. Jones, "Time-resolved characterization of ferroelectrics using high-energy X-ray diffraction”, IEEE Trans. Ultrason. Ferroelectr. 56 (2009) 8.

[36] G. Yueqiu, D. Hui, Z. Xuejun, P. Jinfeng L. Xujun, H. Renjie, "Large piezoelectric response of Bi0.5( $\mathrm{Na}(1-\mathrm{x}) \mathrm{Kx}) 0.5 \mathrm{TiO}$ thin films near morphotropic phase boundary identified by multi-peak fitting", J. Phys. D. Appl. Phys. 45 (2012) 305301.

[37] G. Suchaneck, R. Koehler, P. Padmini, T. Sandner, J. Frey, G. Gerlach, Self-polarization control of radio-frequency-sputtered lead zirconate titanate films, Surf. Coat. Technol. 116 (1999) 1238. 